Revista de BIOLOGía TROPICAL

\title{
Políticas Públicas: Visión general y realidad en el Parque Nacional Isla del Coco, Costa Rica
}

\author{
Mary Luz Moreno-Díaz \\ Centro Internacional de Política Económica para el Desarrollo Sostenible, Universidad Nacional, Heredia, Costa Rica; \\ mmoreno@una.cr
}

Recibido 08-V-2015. Corregido 17-VI-2015. Aceptado 02-VII-2015.

\begin{abstract}
Public Policies: from a general vision to Isla del Coco National Park, Costa Rica. Costa Rica has a set of policies emerged from a process of improvement and analysis of previous instruments and the need to consolidate the management of the Wildlife Protected Areas of the country. In this article, we review the overall framework of public policies, what it means and what it should cover; and finish with a review the overall policy framework of protected areas in Costa Rica with a focus on its application in Isla del Coco National Park. Policies must be improved, including budget, indicators and verification. Rev. Biol. Trop. 64 (Suppl. 1): S277-S290. Epub 2016 Febrary 01.
\end{abstract}

Key words: Isla del Coco National Park, Public Policy, Wild Protected Areas, Policy Cycle, Evaluation, Costa Rica.

El Parque Nacional Isla del Coco, se ubica $535 \mathrm{~km}$. al sur oeste de Cabo Blanco, en el Océano Pacifico, en Costa Rica. El Parque Nacional Isla del Coco (PNIC) fue declarado como tal el 22 de junio de 1978, por medio del Decreto Ejecutivo No. 8748-A, bajo la administración del ex presidente Rodrigo Carazo Odio. El PNIC posee una extensión de 209506 ha: 2347 ha de área terrestre (1.2\%) y 207159 ha de ecosistemas marinos (98.8\%) (Bermúdez-Acuña, Zanella, \& Ballestero, 2007). Es reconocida mundialmente como reservorio de biodiversidad y endemismo, sitio de belleza paisajística excepcional y se considera como uno de los diez mejores sitios para buceo recreativo en el mundo. Por sus características, la Isla del Coco constituye uno de los sitios naturales más privilegiados a nivel mundial.

El PNIC es parte de las 169 áreas silvestres protegidas con que cuenta Costa Rica. En los últimos años se ha venido trabajando en la generación de instrumentos que permitan el adecuado manejo de estas áreas, que garanticen la efectividad en la conservación de los recursos biológicos que albergan. Con este fin se estructuraron las estrategias: "Hacia la Administración eficiente de las Áreas Silvestres Protegidas: Políticas e indicadores", la "Agenda para las Áreas Silvestres Protegidas Administradas por el SINAC" cuya duración se planteó para 10 años (2002-2012). Además se cuenta con el Plan Estratégico Institucional 2010-2015 y el Plan de Acción del SINAC 2013-2017 que se enmarcan en las Políticas para las Áreas Silvestres Protegidas para el 2011-2015.

Una parte fundamental del quehacer del gobierno se refiere al diseño, gestión y evaluación de las políticas públicas. Las políticas públicas son soluciones específicas de cómo manejar los asuntos públicos y se desprenden de la agenda pública. Una parte fundamental del desarrollo de las políticas es la evaluación, la determinación de si se están cumpliendo los objetivos propuestos por la política o no. El presente artículo busca estructurar una base teórica de la formulación, el ciclo y la 
evaluación de las políticas públicas, para luego realizar un análisis de las políticas públicas para ASP en Costa Rica en general y aquellas desarrolladas para el Parque Nacional Isla del Coco en particular.

\section{MATERIALES Y MÉTODOS}

Se desarrolla una revisión teórica de las políticas públicas, lo que significan y lo que deberían contemplar desde la formulación hasta la evaluación. Luego se analizan las políticas públicas para áreas protegidas en Costa Rica, iniciando con la descripción de los marcos legales internacionales (desde 1966), regionales (desde 1991) y nacionales (desde 1909) que se han venido desarrollando y que conforman las bases de las leyes y políticas actuales en estas áreas. Se hace también una reseña de las políticas para áreas protegidas de 1997 y 2003 que no fueron oficializadas y que sirvieron de base para las políticas del 2011-2015 que si se oficializaron y se están aplicando actualmente. Finalmente se analizan algunos de los indicadores e información disponible de los resultados de la política pública para áreas protegidas (2011-2015) en el PNIC.

\section{RESULTADOS}

\section{Base Teórica Conceptual de las Políticas Públicas}

Hay diferentes concepciones de lo que es una política pública. Según Heclo \& Wildavsky (1974), es una "acción gubernamental dirigida hacia el logro de objetivos fuera de ella misma". Para Meny \& Thoening (1992) es "el resultado de una actividad de una autoridad investida de poder público y autoridad gubernamental", y para Tomassini (1998), son: (1) un curso de acción estable (2) definido por el gobierno (3) para resolver un área relevante de asuntos de interés público (4) en cuya definición en las actuales sociedades suelen también participar actores de origen privado.

Subirats, Knoepfel, Larrue, \& Varone (2008), argumentan que "Aunque estas definiciones son muy diversas, todas tienden a acentuar algún factor, sean los actores investidos en los poderes públicos, sean los problemas públicos a resolver, sean las soluciones estatales adoptadas".

Cualquiera que sea el marco teórico en el que se basa la política pública generalmente contienen los siguientes elementos generales (Subirats et al., 2008):

a. Solución de un problema público: Pretende resolver problemas sociales reconocidos políticamente como público.

b. Existencia de grupos-objetivo en el origen de un problema público: Toda política pública busca modificar u orientar la conducta de la población específica, ya sea de manera directa o actuando sobre el entorno de los actores.

c. Una coherencia al menos intencional: Supone que las decisiones y las actividades que se lleven a cabo estén relacionadas entre sí.

d. Existencia de diversas decisiones y actividades: Se caracteriza por un conjunto de acciones que se basan en un nivel de decisiones única o específica.

e. Programa de intervenciones: Ante las decisiones tomadas, se debe contar con decisiones más o menos concretas $\mathrm{e}$ individualizadas.

f. Papel clave de los actores públicos: Se considerara como una política pública en la medida en que quienes las toman y las llevan a cabo lo realizan desde su condición de actores públicos.

g. Existencia de actos formales: Una política pública supone la producción de actos u outputs que pretenden orientar el comportamiento de grupos o individuos que, se supone, originan el problema público a resolver.

h. Naturaleza más o menos obligatoria de las decisiones y actividades: Las formas de accionar pública son actualmente tanto incentivadoras como coercitivas, muchas de las intervenciones públicas se llevan 
a través de un contrato entre el Estado y los administradores.

\section{El ciclo de una política pública}

De acuerdo con Müller (1990) las políticas son representaciones secuenciales que no deben ser empleadas de manera mecánica, deben desarrollarse como un flujo continuo de decisiones y procedimientos a los que se les da un sentido. En la Figura 1 se presenta un ciclo simplificado de una política pública. El (Re) surgimiento de un problema se produce ante una necesidad colectiva, una carencia en una situación existente, a la cual se le busca una solución. La fase de la incorporación a la agenda política, se refiere a un filtro donde los actores decisivos del sistema político, toman en cuenta las demandas sociales (Knoepfel, Kissling-Näf, \& Varone, 2001).

La formulación de la política corresponde a la definición del modelo causal por parte de los actores públicos, los cuales influyen en mayor o menor medida. La fase de implementación consiste en concretar el programa a las situaciones que se va a enfrentar. Y finalmente, la fase de evaluación consiste en la efectividad y los resultados de la política que se implementó, ante los cambios de conducta que se querían llevar a cabo.

Las políticas no implican una secuencia lineal y estable de eventos bajo el comando de diseñadores racionales: hay una gran inestabilidad y gran cantidad de variables que no son controladas por un solo tipo de actor (Moreno, 2009). Las políticas evolucionan en el contexto que se encuentre y por lo tanto son adaptables al mismo.

Los impactos que generan las políticas pueden ser a corto, mediano o largo plazo en cualquiera de los casos, el proceso debe estar orientado hacia la creación de condiciones que garanticen la participación, la confrontación y la negociación de las representaciones e intereses de los actores implicados en todas las etapas de gestión de la política, más que en la imposición de la "política correcta" por parte de algunos de los actores implicados. (Moreno, 2009)

\section{Los actores de las políticas públicas}

Las interacciones de las fases del ciclo de política se realizan con la participación

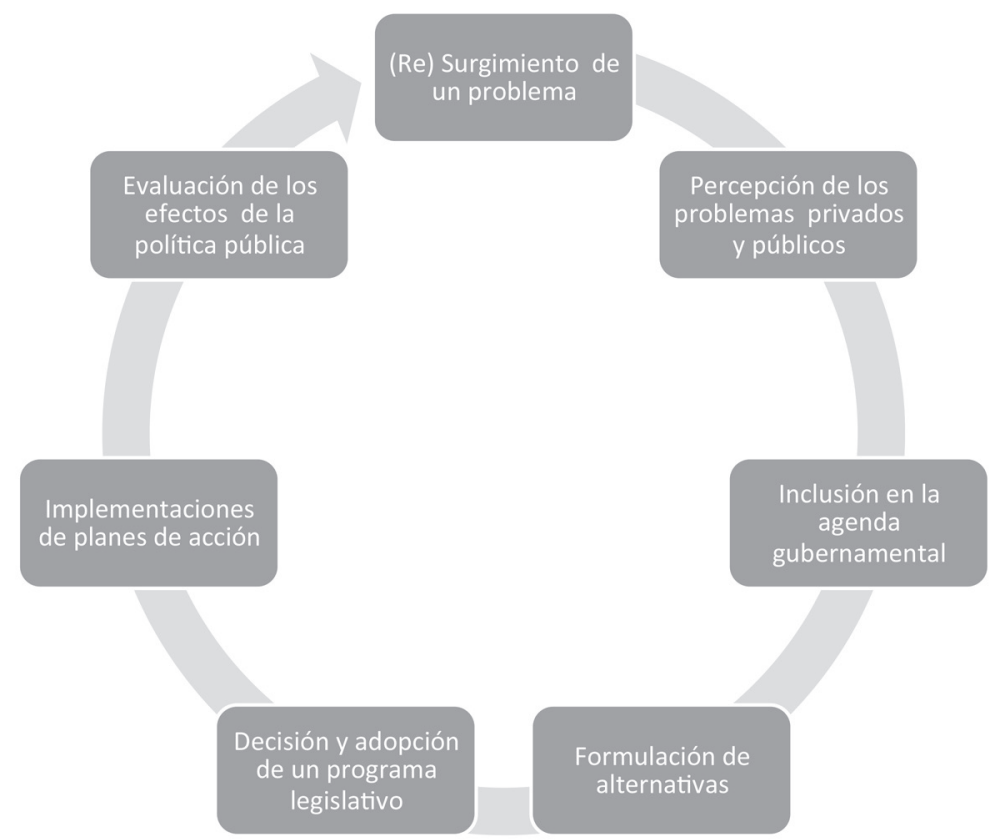

Fig. 1. Ciclo de una Política Pública. Fuente: Parson, 1995. / Fig. 1. The Public Policy cycle. Source: Parson,1995. 


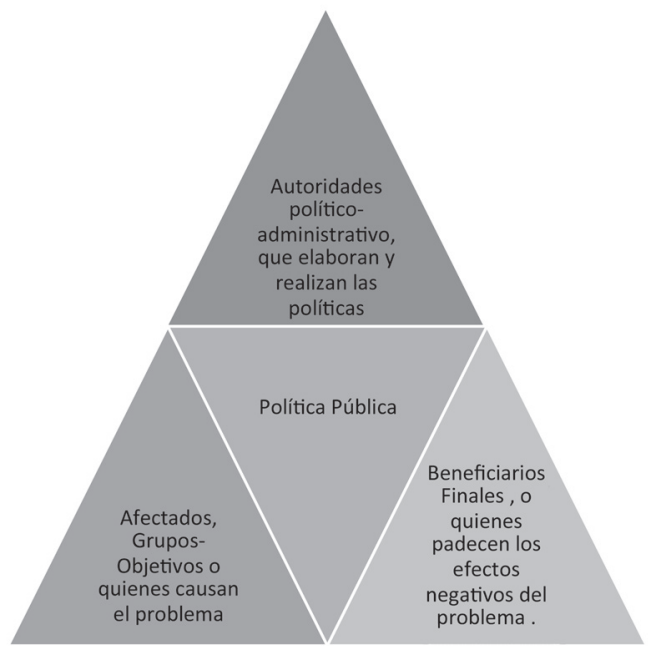

Fig. 2. Tipos de actores en las políticas públicas. Fuente: Knoepfel et al., 2001.

Fig. 2. Actors in public policy. Source: Knoepfel et al., 2001.

de diferentes actores como las autoridades políticas - administrativas, los afectados y los beneficiados, los cuales se interrelacionan en el espacio de una política pública, con los recursos disponibles de cada actor (Fig. 2).
Los actores y las estructuras de poder relevantes están involucrados en la toma de decisiones, para lo cual las políticas no deben ser un proceso de solución racional de problemas, sino de interacción entre múltiples actores con intereses, valores y visiones divergentes (Moreno, 2009).

\section{Los diferentes tipos de recursos}

La relación de los actores y los recursos presenta un peso relativo, ya que los recursos pueden variar de una situación a otra, y la presencia de intercambio de recursos se realiza con el fin de cumplir con los objetivos, cada uno de los recursos se expresan en la Figura 3.

\section{Las Políticas Públicas para Áreas Silvestres Protegidas en Costa Rica}

Las políticas públicas desarrolladas para ASP en Costa Rica deberían seguir, en principio, los aspectos contemplados en el ciclo de la política, teniendo en cuenta los actores y diferentes tipos de recursos analizados en las secciones anteriores. A continuación se

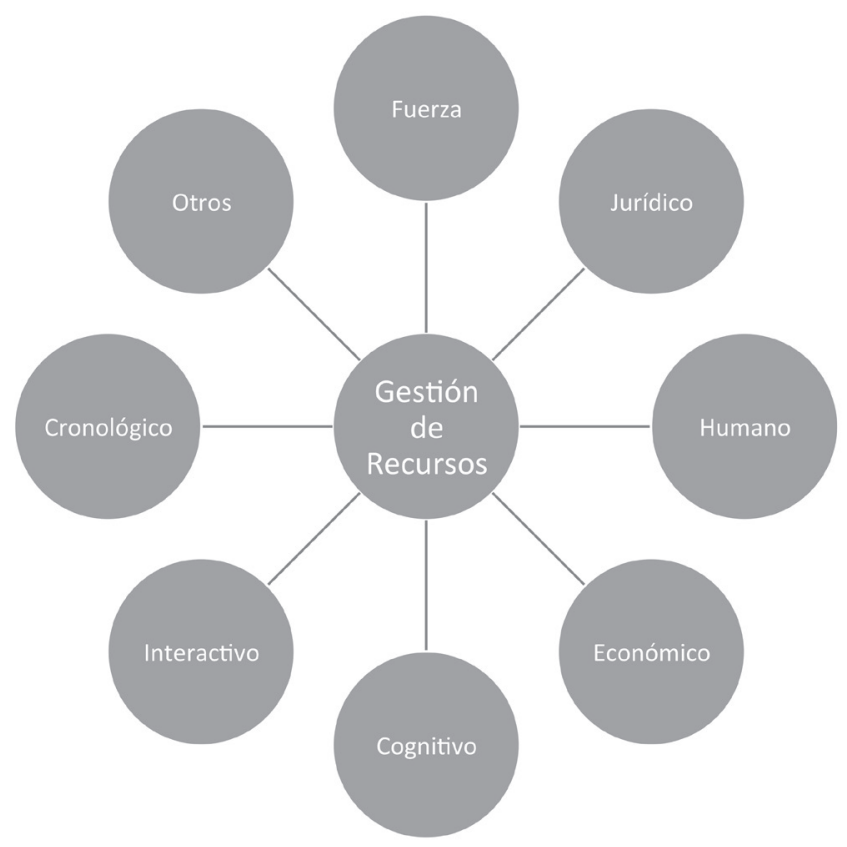

Fig. 3. Tipos de recursos de una Política Pública. Fuente: Knoepfel et al., 2001.

Fig. 3. Public Policy resources. Source: Knoepfel et al., 2001. 
hace una breve descripción de lo que han sido estas políticas.

El conocimiento generado durante más de 50 años desde la creación del primer parque nacional y con la consolidación del Sistema Nacional de Áreas de Conservación, ha obligado al país a mantenerse en un constante proceso de innovación en la gestión que realiza de sus 169 Áreas Silvestres Protegidas. La experiencia desarrollada ha permitido mejorar constantemente el marco jurídico, organizacional $\mathrm{y}$ administrativo en materia ambiental y buscando adecuarlo a las exigencias del momento.

Antes del 2010 Costa Rica no disponía de una política pública para Áreas Silvestres Protegidas, aunque empleaba algunos documentos como "Políticas para Áreas Silvestres Protegidas de Costa Rica" de 1997 y "Políticas de protección y control" de 1998, pero ninguno de estos habían sido oficializados ni formalmente divulgados (CGR, 2008). Debido a lo anterior, la contraloría General de la republica dispuso que el director Ejecutivo del SINAC tenía que establecer y promulgar el procedimiento para la emisión de políticas, estrategias y lineamientos que orienten la gestión institucional.

Fue así que el SINAC, que contaba además con un conjunto de políticas en el documento "Hacia la administración Eficiente de las Áreas Silvestres Protegidas: Políticas e indicadores para su monitoreo" del 2003, elaboró y publicó las "Políticas para las Áreas Silvestres Protegidas del Sistema Nacional de Áreas de Conservación (SINAC) 2011-2015. Costa Rica" en el 2010, cuyo objetivo es el de "Consolidar un sistema de Áreas Silvestres Protegidas para la conservación in situ, que sea comprensivo, eficazmente gestionado y ecológicamente representativo de la diversidad biológica del país, por medio del reconocimiento, promoción y fortalecimiento de los diferentes modelos de gobernanza que garanticen la provisión a largo plazo de bienes y servicios ecosistémicos" (SINAC, 2010). En este documento se especificaron 9 políticas para las ASP para el periodo comprendido entre 2011 a 2015 (Cuadro 1).

Posteriormente se creó la Política Nacional del Mar Costa Rica (PNM) 2013-2028, que

CUADRO 1

Políticas para las Áreas Silvestres Protegidas. Costa Rica. 2011-2015

TABLE 1

Policies for Wild Protected Areas. Costa Rica. 2011-2015

1. Representatividad ecológica: El sistema nacional de áreas silvestres protegidas debe contar con muestras representativas de cada uno de los ecosistemas naturales más relevantes que tengan presencia en el territorio continental, insular y marino del país, así como garantizar la conectividad que permitan mantener y recuperar la integralidad de los ecosistemas. Cuenta con dos objetivos y seis lineamientos.

2. Participación pública: La gestión de las ASP incorporará instrumentos y mecanismos de participación pública que permitan la aplicación de diferentes modelos de gobernanza en la gestión de las ASP. A la vez, promoverá en la población, la capacidad de incidir en la toma de decisiones y políticas públicas y su incorporación como sujetos activos de la conservación y protección de la biodiversidad. Cuenta con un objetivo y seis lineamientos

3. Turismo sostenible: La gestión del turismo en las ASP deberá desarrollarse dentro de un marco de sostenibilidad, integrado con sus áreas de influencia y articulado con las políticas, planes y programas nacionales de conservación y turismo. Cuenta con un objetivos y cinco lineamientos

4. Patrimonio Natural del Estado (PNE) en ASP: Consolidar la propiedad y ejercer el dominio sobre los terrenos que conforman el patrimonio natural del Estado, de acuerdo con su categoría de manejo. Cuenta con un objetivo y tres lineamientos.

5. Manejo, control y protección de las ASP: Establecer un sistema de gestión de ASP sustentada en criterios científicos de manejo, enfatizando en la prevención de daños y con sistemas de control eficaces y eficientes que garanticen la conservación de la biodiversidad. Cuenta con un objetivo y nueve lineamientos.

6. Gestión del conocimiento: La gestión de las ASP estará sustentada en una sólida base de conocimiento científico, técnico y tradicional que permita el mejoramiento continuo y mayores niveles de eficacia y eficiencia en la conservación de la biodiversidad. Cuenta con un objetivo y seis lineamientos 
7. Ordenamiento territorial y espacial marino: El ordenamiento territorial en los niveles nacional y regional y en los planes reguladores locales debe incorporar criterios e indicadores ambientales que tomen en cuenta elementos de conectividad y conservación de las ASP. Cuenta con un objetivos y cinco lineamientos

8. Respuesta ante amenazas globales y locales: El cambio climático debe de ser un eje transversal en todas las acciones de conservación in situ, valorando y tomando en cuenta en especial, el rol que las ASP juegan en la mitigación y adaptación al cambio climático para mejorar la gestión y de esa manera reducir la vulnerabilidad. Cuenta con un objetivo y cuatro lineamientos

9. Capacidad institucional: El crecimiento continuo de la capacidad institucional orientará todos los procesos técnicos, administrativos y directivos que guían la gestión de las ASP, y se sustentará en procesos permanentes de capacitación y de mejora de las condiciones de los funcionarios que laboran en las ASP. Cuenta con cuatro objetivos y 21 lineamientos

Fuente: SINAC 2010.

para algunas ASP puede ser complementaria con la Política específica para estas áreas. Sin embargo el presente artículo se concentra en la Política para ASP, ya que la PNM todavía no ha entrado en fase de implementación.

Las políticas para ASP se enmarcan en una estructura institucional partiendo de que se describe a continuación:

\section{Marco Legal}

Costa Rica cuenta con un marco regulador en material ambiental muy heterogéneo, debido a la complejidad en la protección de la biodiversidad. A pesar de las circunstancias Costa Rica posee convenios regionales, internacionales (en el momento de ser ratificados se convierten en ley a nivel nacional) y leyes nacionales, entre otros mecanismos de protección ambiental.

En el artículo 6 de la Ley de Administración Pública se establece la jerarquía de las fuentes del ordenamiento jurídico administrativo (Fig. 4).

En el artículo 50 de la Constitución Política de Costa Rica se establece "Toda persona tiene derecho a un ambiente sano y ecológicamente equilibrado. Por ello, está legitimada para denunciar los actos que infrinjan ese derecho y para reclamar la reparación del daño causado. El Estado garantizará, defenderá y preservará ese derecho." Este artículo fue reformado mediante Ley 7412 el 24 de mayo de 1994.

\section{Ámbito Internacional}

Los Convenios internacionales son considerados como acuerdos escritos entre las partes en la búsqueda del derecho a nivel internacional. Los convenios son instrumentos de resolución de conflictos entre las partes o el respeto hacia un tema de interés internacional. Los involucrados deben de actuar de acuerdo al convenio establecido y firmado. (Cajiao, 2008).

Según Moreno, González, \& Mora (2010) y SINAC (2012) en Costa Rica el SINAC es punto focal de convenios internacionales como mecanismos de protección de la biodiversidad. Algunos convenios y convenciones importantes en materia ambiental son:

1. Convención para la protección de la flora, fauna y de las bellezas escénicas naturales de los países de América. Ley N 3763. Publicado en 1966.

2. Convenio sobre el Comercio Internacional de Especies Amenazadas de fauna y flora silvestres (CITES) Ley No 5605. Ratificado en 1975.

3. Convención sobre Humedales Internacionales como Hábitat de Aves Acuáticas (RAMSAR). Ley No 7224. Ratificado en 1991.

4. Convenio para la protección y desarrollo del medio marino y su protocolo para combatir derrames de Hidrocarburos en la región del Gran Caribe. Ley N 7227. Publicado en 1995. 


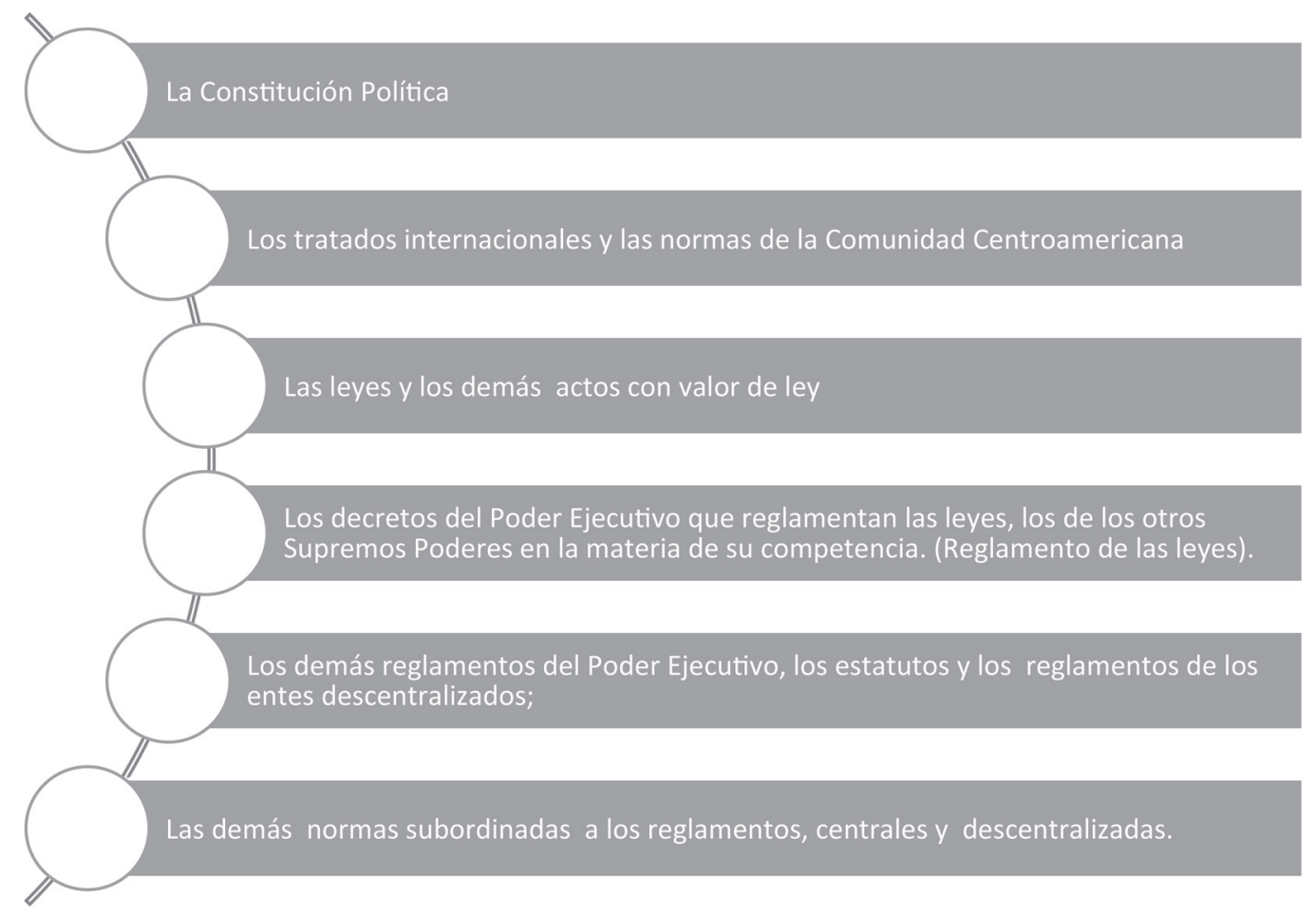

Fig. 4. Jerarquía de las fuentes del ordenamiento jurídico administrativo en Costa Rica. Fuente: Ley No. 6227, Ley General De La Administración Pública.

Fig. 4. Hierarchy of sources of administrative law in Costa Rica. Source: Law No. 7227, General Law of Public Administration of Costa Rica.

5. Convenio sobre Diversidad Biológica (CDB). Ley $\mathrm{N}^{\circ}$ 7416. Ratificado en 1994.

6. Convención de las Naciones Unidas sobre el Derecho del Mar (CONVEMAR). Entró en vigor el 16 de noviembre de 1994.

7. Convención de las Naciones Unidas sobre el derecho del mar. Ley $N^{\circ} 7291$. Ratificado en 1992.

8. Convención Marco de las Naciones Unidas Sobre el Cambio Climático. Ley $\mathrm{N}^{\circ}$ 7414. Ratificado en 1992.

9. Protocolo de Montreal relativo a sustancias agotadoras de la capa de ozono. Ley $\mathrm{N}^{\circ}$ 7223. Ratificado en 1995.

10. Convención sobre la Conservación de Especies Migratorias de Animales Silvestres (CMS). Ley $N^{\circ}$ 8586. Ratificado en el 2007.
11. Convención Interamericana de Tortugas Marinas. Ley $\mathrm{N}^{\circ}$ 7906. Fecha de Promulgación 1999.

12. Convenio de Viena para la Protección de la Capa de Ozono. Ley $\mathrm{N}^{0}$ 7228. Ratificado en 1991.

13. Convención de lucha contra la desertificación y degradación de Tierras (UNCCD). Ley Nº7699. Fecha de Promulgación 1997.

14. Aprobación del convenio internacional sobre responsabilidad civil nacida de daños debidos a contaminación por hidrocarburos y sus protocolos. Ley N ${ }^{0} 7627$. Fecha de Promulgación 1996.

\section{Ámbito Regional}

1. Convenio para la protección y desarrollo del medio marino y su protocolo para 
combatir derrames de hidrocarburos en la región del Gran Caribe y su Protocolo. Ley $\mathrm{N}^{\circ}$ 7227. Fecha de promulgación 1991.

2. Convenio constitutivo de la Comisión Centroamérica de Ambiente y Desarrollo y su protocolo. Ley $\mathrm{N}^{\mathrm{o}} 7226$. Ratificado en 1991.

3. Convenio para la conservación de la biodiversidad y protección de áreas silvestres prioritarias en América Central. Ley $\mathrm{N}^{\circ}$ 7433. Ratificado en 1994.

4. Convenio regional para el manejo y conservación de ecosistemas naturales forestales y del desarrollo de plantaciones forestales, conocido como Convenio Centroamericano de Bosques. Ley $\mathrm{N}^{\circ} 7572$. Ratificado en 1996.

5. Convenio para la conservación de la biodiversidad y protección de las áreas silvestres prioritarias en América Central. Ley $\mathrm{N}^{\mathrm{o}}$ 7433. Ratificado en 1994.

6. Convención para la protección de la flora, la fauna y las bellezas naturales de los países de América. Se convirtió en la Ley $\mathrm{N}^{\mathrm{o}}$ 3763. Fue firmado en el año 1940 y ratificado en Costa Rica en 1966.

7. Acuerdo entre el Gobierno de Costa Rica y la Unión Internacional para la Conservación de la Naturaleza y los Recursos Naturales (U.I.C.N), para el Establecimiento de su Oficina Regional para Centroamérica, suscrito en la Ciudad de San José. Ley N ${ }^{\circ}$ 7350. Fecha de promulgación 1993.

8. Convenio Centroamericano Regional sobre cambios climáticos. Ley $\mathrm{N}^{\mathrm{o}} 7513$. Ratificado en 1995.

9. Acuerdo Regional Centroamericano sobre movimiento transfronterizo de desechos peligrosos. Ley $\mathrm{N}^{\circ}$ 7520. Ratificado en 1995.

10. Alianza para el Desarrollo Sostenible en Centroamérica (ALIDES). 1994.

11. Aprobación del Convenio de las naciones unidas sobre el derecho del mar. Ley $\mathrm{N}^{\mathrm{o}}$ 7291. Fecha de promulgación1992.

12. Convenio sobre la Diversidad Biológica y sus anexos i y ii firmado el 13 de junio de 1992 en Río de Janeiro, Brasil. Ley N ${ }^{\circ}$ 416. Fecha de promulgación 1994.

13. Aprobación del tratado sobre delimitación de áreas marinas y submarinas y cooperación marítima entre la República de Costa Rica y la República de Colombia. Ley $\mathrm{N}^{\circ}$ 8084. Fecha de promulgación 2001.

\section{Ámbito Nacional}

Costa Rica en la protección de los recursos naturales y la biodiversidad cuenta con una amplia normativa jurídica en materia ambiental, aproximadamente se encuentran unas 121 leyes (SINAC, 2012). Algunas leyes relacionadas con ASP y la biodiversidad se citan en el Cuadro 2.

En cuanto al marco organizacional, las competencias alrededor de las ASP se desarrollan de la siguiente manera:

Ministerio del Ambiente y Energía (MINAE), es el encargado de "dictar políticas, planificar y ejecutar procesos dirigidos a lograr la sostenibilidad en el manejo de los recursos naturales de Costa Rica" (Ley de Biodiversidad.1998. Art 22)

Sistema Nacional de Áreas Protegidas (SINAC). El SINAC "será un sistema de gestión y coordinación institucional, desconcentrado y participativo, que integrará las competencias en materia forestal, vida silvestre, áreas protegidas" (Ley de Biodiversidad.1998. Art 22). Las Áreas Silvestres Protegidas forman parte de la estructura organizativa del Sistema Nacional de Áreas de Conservación (Fig. 5). El SINAC está conformado por 1) El Consejo Nacional de Áreas de Conservación (CONAC), 2) La Secretaría Ejecutiva, 3) Las estructuras administrativas de las Áreas de Conservación, 4) Los Consejos Regionales de Áreas de Conservación y 5) Los Consejos Locales.

Consejo Nacional de Áreas de Conservación (CONAC). De acuerdo a la Ley de Biodiversidad en su artículo 25 las funciones de este órgano son:

- Definir estrategias y políticas relacionadas con la consolidación y el desarrollo de las 
CUADRO 2

Leyes relacionadas con Áreas Silvestres Protegidas y Biodiversidad de Costa Rica

TABLE 2

Laws related to Protected Areas and Biodiversity in Costa Rica

\begin{tabular}{llr}
\multicolumn{1}{c}{ Ley } & \multicolumn{1}{c}{ Titulo } & Fecha \\
121 & Ley de cercas divisorias y quemas & 1909 \\
276 & Ley de aguas & 1942 \\
190 & Ley de pesca y caza marítimas & 1948 \\
8325 & Ley de protección, conservación y recuperación de las poblaciones de tortugas marinas. & 1949 Rev.1999 \\
6043 & Ley sobre la Zona Marítimo Terrestre & 1977 \\
6084 & Ley de Creación del Servicio de Parques Nacionales & 1977 \\
6919 & Ley de Conservación de la Fauna Silvestre & 1983 \\
7317 & Ley de Conservación de la Vida Silvestre & 1992 \\
7554 & Ley Orgánica del Ambiente & 1995 \\
7575 & Ley Forestal 7575 & 1996 \\
7788 & Ley de Biodiversidad & 1998 \\
8133 & Reformas al inciso a) del artículo 3 de la Ley No. 5100 y sus reformas, y creación de la Junta & 2001 \\
& Directiva del Parque Recreativo Nacional Playas de Manuel Antonio. & 2005 \\
\hline
\end{tabular}

áreas protegidas estatales, así como supervisar su manejo.

- Aprobar las estrategias, la estructura de los órganos administrativos de las áreas protegidas y los planes y presupuestos anuales de las Áreas de Conservación.

- Recomendar la creación de nuevas áreas protegidas que aumenten su categoría de protección.

- Realizar auditorías técnicas y administrativas para la vigilancia del buen manejo de las Áreas de Conservación y sus áreas protegidas.

\section{Áreas de Conservación}

El Sistema Nacional de Áreas de Conservación se encuentra estructurado en Áreas de Conservación (AC) supervisados por los entes ya mencionados, entonces se denomina AC como "una unidad territorial del país, delimitada administrativamente, regida por una misma estrategia de desarrollo y administración, debidamente coordinada con el resto del sector público. En cada uno se interrelacionan actividades tanto privadas como estatales en materia de conservación sin menoscabo de las áreas protegidas" (Ley de Biodiversidad. 1998. Art 28). El total de las AC son:

- Área de Conservación Arenal Huetar-Norte (ACA-HN)

- Área de Conservación Arenal Tempisque (ACAT)

- Área de Conservación Amistad Caribe (ACLA-C)

- Área de Conservación Amistad Pacífico (ACLA-P)

- Área de Conservación Cordillera Volcánica Central (ACCVC)

- $\quad$ Área de Conservación Guanacaste (ACG)

- Área de Conservación Marina Isla del Coco (ACMIC)

- Área de Conservación Osa (ACOSA)

- Área de Conservación Pacífico Central (ACOPAC)

- Área de Conservación Tempisque (ACT)

- Área de Conservación Tortuguero (ACTo).

Áreas Silvestres Protegidas

De acuerdo a las consideraciones anteriores las áreas protegidas se encuentran dentro de las AC (Fig. 5). En Costa Rica las 


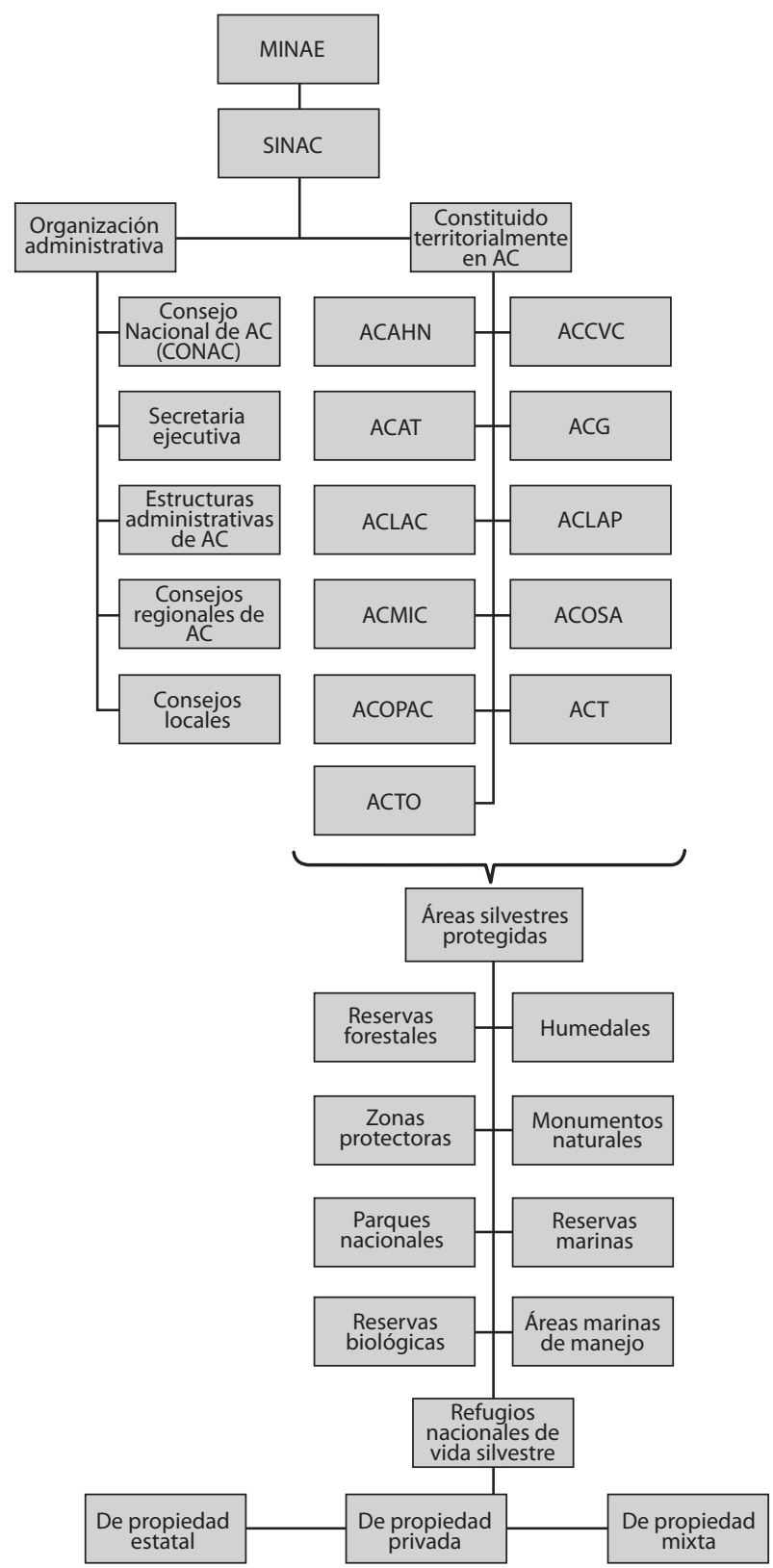

Fig. 5. Marco organizativo alrededor de las Áreas Silvestres Protegidas. Fuente: Elaboración propia con información de la Ley de Biodive rsidad N7788 y el Reglamento a la Ley de Biodiversidad.

Fig. 5. Organizational frame around the Wildlife Protected Areas. Source: Based on information from the Biodiversity Law No. 7788 and the Regulation of the Law of Biodiversity.

Áreas Silvestres Protegidas se definen como: "Espacio geográfico definido, declarado oficialmente y designado con una categoría de manejo en virtud de su importancia natural, cultural y/o socioeconómica, para cumplir con determinados objetivos de conservación y de gestión." (Reglamento a la Ley de Biodiversidad, 2008. Art. 3). Y como "zonas geográficas delimitadas, constituidas por terrenos, humedales y porciones de mar. Han sido declaradas 
como tales por representar significado especial por sus ecosistemas, la existencia de especies amenazadas, la repercusión en la reproducción y otras necesidades y por su significado histórico y cultural. Estas áreas estarán dedicadas a conservación y proteger la biodiversidad, el suelo, el recurso hídrico, los recursos culturales y los servicios de los ecosistemas en general" (Ley de Biodiversidad, 1998. Art 58).

Las ASP se subdividen en categorías de manejo establecido en el artículo 32 de la Ley Orgánica, se señalan a continuación:

- Reservas Forestales.

- Zonas Protectoras.

- Parques Nacionales.

- Reservas Biológicas.

- Refugios Nacionales de Vida Silvestre: se clasifican en tres clases:

- Refugios Nacionales de Vida Silvestre de propiedad estatal.

- Refugios Nacionales de Vida Silvestre de propiedad privada.

- Refugios Nacionales de Vida Silvestre de propiedad mixta.

- Humedales.

- Monumentos Naturales.

- Reservas Marinas.

- Áreas Marinas de Manejo.

- Administrativo

\section{Recursos Financieros y Humanos}

Para el cumplimiento de las políticas públicas en ASP, el SINAC cuenta con varias fuentes de ingreso: el Presupuesto Ordinario, el Fondo de Parques Nacionales, el Fondo Forestal, el Fondo de Vida Silvestre, el Programa de Pago por Servicios Ambientales y otros.

\section{Estudio de Caso: EI Parque Nacional Isla del Coco (PNIC)}

El Parque Nacional Isla del Coco (PNIC) se encuentra ubicado en el Área de Conservación Marina isla del Coco (ACMIC) en la que también se creó en el 2011 el Área Marina de Manejo de Montes Submarinos (AMM-MS), que no será objeto de estudio en este artículo.
El PNIC, es una de las 160 áreas silvestres protegidas de Costa Rica; actualmente incluye a la Isla del Coco, los islotes aledaños y un área de 12 millas náuticas alrededor de la isla, posee una superficie de $24 \mathrm{~km}^{2}$. Fue reconocida por la UNESCO como Patrimonio Mundial de la Humanidad en 1997. Es reconocida mundialmente como reservorio de biodiversidad y endemismo, sitio de belleza paisajística excepcional y se considera como uno de los diez mejores sitios para buceo recreativo en el mundo. Por sus características, la Isla del Coco constituye uno de los sitios naturales más privilegiados a nivel mundial. (ACMIC, 2009).

\section{Implementación de las políticas al caso del Parque Nacional Isla del Coco}

Hasta el 2010 el PNIC contaba con una serie de instrumentos en los que se enmarcaba su gestión:

- $\quad$ Plan Estratégico ACMIC 2005-2020.

- El plan de Manejo del PNIC desarrollado en el 2007.

- Plan de Monitoreo del Impacto del Turismo Marino en el Parque Nacional Isla del Coco.

- Reglamento de Uso Público del Parque Nacional Isla del Coco. Aprobado en sesión de CONAC del 29 de Noviembre del 2010. Publicado. Alcance Digital No 62 del 10 de mayo del 2012.

- Los diferentes programas:

- Programa de Administración y Operaciones. Se encarga de la gestión administrativa del Parque.

- Programa de Turismo Sostenible. Es el responsable del desarrollo de la actividad turística en el Parque.

- Programa de Control y Vigilancia. Dicho programa vela por el control de la pesca ilegal.

Posterior al 2010 además de estos instrumentos el PNIC cuenta con la Política Nacional para ASP 2011-2015 y con otros estudios como: 
- Herramienta para la evaluación de la efectividad de manejo de las Áreas Silvestres Protegidas de Costa Rica. Biomarc, 2014.

- Investigaciones y proyectos desarrollados por otras organizaciones no públicas que generan insumos valiosos para el desarrollo de las políticas. Con relación a las publicaciones, Cortes (2012), realizó en el 2012 una revisión bibliográfica en la que encontró 599 referencias de artículos científicos y capítulos de libros del PNIC, además de 41 tesis, libros, volúmenes especiales, memorias de reuniones, informes sobre investigaciones marinas, oceanográficas, geológicas y atmosféricas, un sitio web y diez libros históricos en los que se mencionan a organismos y ambientes marinos de la Isla del Coco.
- Es importante mencionar también que Moreno (2012) desarrolló un estudio en el cual aproximó la contribución socioeconómica del PNIC para el 2010 en 8,3 millones de dólares.

- Estadísticas consignadas en CEMEC. En el Cuadro 3 se resumen las estadísticas para 2011 al 2014 y se relacionan con las diferentes políticas especificadas para las ASP

\section{Dificultad en la aplicación de las Políticas}

Como se mencionó en el marco teórico del presente artículo parte importante del ciclo de políticas es la evaluación de los efectos de las mismas, de este modo es posible analizar si se alcanzaron los objetivos propuestos, con la

CUADRO 3

Estadísticas sobre la gestión del PNIC y relación con las Políticas Públicas

TABLE 3

Statistics related to the PNIC management and relationship with Public Policy

\begin{tabular}{|c|c|c|c|c|c|}
\hline Categoría & 2011 & 2012 & 2013 & 2014 & $\begin{array}{l}\text { Responde a } \\
\text { la política }\end{array}$ \\
\hline $\begin{array}{l}\text { Actividades de educación ambiental. } \\
\text { \# de beneficiados de las charlas }\end{array}$ & 4531 & 2865 & ND & ND & 2 \\
\hline Voluntariado & 128 & 288 & 65 & 69 & 2 \\
\hline Visitantes & 2534 & 3131 & 2489 & 2481 & 3 \\
\hline $\begin{array}{l}\text { Cantidad de denuncias interpuestas } \\
\text { en tribunales }\end{array}$ & 5 & 24 & 19 & 8 & 5 \\
\hline $\begin{array}{l}\text { Cantidad de actividades de control y } \\
\text { protección efectuadas }\end{array}$ & $\begin{array}{l}34854 \text { horas } \\
\text { hombre dedicadas } \\
\text { a actividades de } \\
\text { control y protección }\end{array}$ & $\begin{array}{c}32 \text { (patrullajes, } \\
\text { operativos, quejas } \\
\text { y denuncias) }\end{array}$ & $\begin{array}{c}10 \text { (patrullajes, } \\
\text { operativos, quejas } \\
\text { y denuncias) }\end{array}$ & $\begin{array}{l}24 \text { (patrullajes, } \\
\text { operativos, quejas } \\
\text { y denuncias) }\end{array}$ & 5 \\
\hline Artes de pesca decomisadas & 22 & 98 & 32 & 60 & 5 \\
\hline $\begin{array}{l}\text { Cantidad de permisos de } \\
\text { investigación }\end{array}$ & 11 & 9 & 12 & 10 & 6 \\
\hline Publicaciones de información & $\begin{array}{l}7300 \text { (relacionadas } \\
\text { con Biodiversidad) }\end{array}$ & - & ND & ND & 6 \\
\hline Número de funcionarios & 32 & 33 & 34 & 31 & 9 \\
\hline Presupuesto asignado (colones) & - & 639945422 & 732654292 & 572664680 & \\
\hline Capacitaciones a funcionarios & $\begin{array}{c}48 \text { (Salud, } \\
\text { administración } \\
\text { de ASP, recursos } \\
\text { humanos, calidad } \\
\text { de gestión) }\end{array}$ & $\begin{array}{l}31 \text { (Salud y apoyo } \\
\text { administrativo) }\end{array}$ & $\begin{array}{c}22 \text { (apoyo } \\
\text { administrativo) }\end{array}$ & $\begin{array}{l}5 \text { (planificación y } \\
\text { Biología) }\end{array}$ & 9 \\
\hline Desarollo de infraestructura & 19 & No se desarrolló & $\begin{array}{l}3 \text { (mantenimiento } \\
\text { y otros) }\end{array}$ & No se desarrolló & 9 \\
\hline
\end{tabular}

Fuente: Elaboración propia con en SINAC (2015). 
finalidad de rendir cuentas, hacer un informe presupuestario y realizar un reordenamiento de las políticas de ser necesario. Con lo anterior se constata la eficacia y eficiencia de las políticas empleadas. Es necesario para que los encargados de aplicar las políticas valoren si es necesaria la interrupción de un programa, por ineficiente y valoren otras alternativas. Entre otras decisiones que deben tomar. El principal desafío para llevar a cabo evaluaciones eficaces de impacto es identificar la relación causal entre el proyecto, el programa o la política y los resultados de interés.

En el caso del desarrollo de las políticas para ASP en general y en el PNIC en particular, aunque existe un conjunto de información generada y que pueden ser asociadas a las diferentes políticas no se especifica desde la formulación de las mismas los indicadores y los medios de verificación para el cumplimiento de cada una de los objetivos y sus lineamientos.

Las principales limitaciones identificadas para la aplicación de las políticas en el PNIC son:

- Desde el punto de vista de la pertinencia. Se estipulan las políticas para todas las áreas silvestres protegidas sin identificar sus diferencias. En el caso del PNIC al ser un área marina hay algunos de los objetivos de las políticas no van a poder ser aplicados, como el objetivo 2 de la política 1 .

- Desde el punto de vista del presupuesto. La aplicación de las políticas debe ir acompañado de una contraparte financiera que permita que cada uno de los objetivos de cada política pueda ser desarrollado. En cada uno de los lineamientos se deben generar actividades que tienen un costo $\mathrm{y}$ que debe ser incluido esto no se incluye en el planteamiento de políticas. Es importante mencionar en este punto que en el Plan Estratégico del ACMIC 2005-2020 se identificó como una de las debilidades la frágil situación financiera del ACMIC, que no permite atender las demandas de conservación y protección requeridas.
- Desde el punto de vista de la planificación. El desarrollo de cada una de las políticas debe ser planificada para un periodo determinado de conformidad con la disponibilidad de recursos tanto humanos como financieros dependiendo de la situación de cada una de las Áreas de Conservación.

- Desde el punto de vista de la Evaluación. Para proceder a la evaluación del desarrollo de las políticas 2011-2015, se debe disponer de diferentes indicadores relacionados con cada una de las políticas que como se mencionó anteriormente no se encontró que estén claramente identificados.

\section{DISCUSIÓN}

Las políticas públicas en general siguen un ciclo que inicia con la formulación y concluye en la evaluación de las mismas. Existe un gran desafío para llevar a cabo evaluaciones eficaces ya que se debe identificar la relación causal entre la política y los resultados de interés. Para lo anterior se debe ser muy exhaustivos a la hora de la formulación de la política y dejar estructurado el sistema de evaluación de las políticas para que de acuerdo con una línea base inicial se pueda sistematizar el efecto real que produce la política. Las políticas para Áreas Silvestres Protegidas en Costa Rica se especificaron como un marco general con objetivos y lineamientos que conducirán a la mejor gestión de los recursos de la biodiversidad contenidos en estas Áreas, teniendo en cuenta aspectos socioeconómicos. Sin embargo en la formulación de estas políticas no se especificó un sistema que permitiera evaluar la eficacia de estas políticas una vez concluido su periodo de desarrollo en el 2015. Además de lo anterior, al ser cada Área Silvestre Protegidas tan particular, como es el caso del PNIC, se debe realizar una planificación pormenorizada de cómo se van a aplicar las políticas y sus lineamientos indicando la pertinencia de las mismas en el área específica, los recursos disponibles (presupuestarios y humanos), los plazos de cumplimiento y los indicadores que se generaran para verificar el cumplimiento. 


\section{RESUMEN}

Costa Rica cuenta con un grupo de políticas, que surgió de un proceso de mejora y análisis de instrumentos previos y ante la necesidad de consolidar la gestión de las Áreas Silvestres Protegidas del país. En el presente artículo se hace una revisión del marco general de las políticas públicas, lo que significan y lo que deberían contemplar, para luego revisar el marco general de políticas de las áreas protegidas en Costa Rica, enfocándose en su aplicación en el Parque Nacional Isla del Coco. Las políticas deben ser mejoradas, incluyendo presupuesto, indicadores y medios de verificación.

Palabras clave: Parque Nacional Isla del Coco, Políticas Públicas, áreas silvestres protegidas, Ciclo de Políticas, Evaluación, Costa Rica.

\section{REFERENCIAS}

ACMIC. (2009). Parque Nacional Isla del Coco. Recuperado de http://www.isladelcoco.go.cr/es/areas-silvestres-protegidas.aspx

Bermúdez-Acuña, F., Zanella, I., \& Ballestero, F. (2007). Plan de uso público del Parque Nacional Isla del Coco. San José, Costa Rica: Onca Natural/MINAE/ SINAC.

Cajiao, M.V. (2008). Aspectos legales relacionados con el Parque Nacional Isla del Coco, Costa Rica. Revista de Biología Tropical, 56(Suplemento 2.), 207-214.

Contraloría General de la Républica (CGR). (2008). Sobre la gestión del MINAET en las Áreas Silvestre Protegidas Costeras del País (Informe FOE-PPGAA-0888). San José, Costa Rica: Contraloría general de la República.

Cortés, J. 2012. Bibliografía sobre investigaciones marinas, oceanográficas, geológicas y atmosféricas en el Parque Nacional Isla del Coco y aguas adyacentes, Pacífico de Costa Rica. Revista de Biología Tropical, 60(Suplemento 3), 363-392.

Heclo, H., \& Wildavsky, A. (1974). The Private Goverment of Public Money. Community and Policy Inside British Politcs. Londres: Macmillan.

Knoepfel, P., Kissling-Näf, I., \& Varone, F. (2001). Environmental Policies 1982-2000. Bâle: Helbing \& Lichtenhahn.
Ley de Biodiversidad No. 7788, Diario Oficial La Gaceta 101 (1998).

Ley General de administración Pública No. 6227, Diario Oficial La Gaceta 102 (1978).

Müller, P. 1990. Les politiques publiques. París, Francia: Presses Universitaires de France, Que Sais-je?

Moreno, M. (2009). Proceso y tensiones en la formación de políticas. Presentación en el Curso Planificación y Gestión Estratégica para las Políticas Públicas. Santa Cruz, Bolivia.

Moreno, M. L. (2012). Actividades socioeconómicas en el Parque Nacional Isla del Coco. Costa Rica y posibles efectos de la variabilidad climática. Revista de Biología Tropical, 60(Suplemento 3), 113-129.

Moreno, M. L., González, S., \& Mora, C. (2010). Análisis de la contribución de los parques nacionales y reservas biológicas al desarrollo económico y social a nivel nacional en Costa Rica. (Documento Técnico). Heredia, Costa Rica: UNA, CINPE, SINAC.

Meny, Y., \& Thoening, J. C. (1992). Las Políticas Pública. Barcelona, España: Ariel.

Parson, W. (1995). Public Policy, An Introduction to the Theory and Practice of Policy Analysis. Cheltenham: Edward Elgar.

Reglamento a la ley de Biodiversidad No. 34433, Presidencia de la República (1998).

SINAC. (2010). Políticas para las Áreas Silvestres Protegidas. Documento técnico. SINAC. San José, Costa Rica: MINAE.

SINAC. (2011). SINAC en Números: Informe Década Estadísticas SEMEC 2011. San José, Costa Rica: MINAE.

SINAC. (2012). Memoria Anual Institucional SINAC-2011. San José, Costa Rica: MINAE.

SINAC. (2015). SINAC en Números: Informe Anual Estadísticas SEMEC San José, Costa Rica: MINAE. Recuperado de http://www.sinac.go.cr/documentacion/Paginas/Transparencia.aspx

Subirats, J., Knoepfel, P., Larrue, C., \& Varone, F. (2008). Analisis y gestión de políticas públicas. Barcelona, España: Ariel.

Tomassini, L. (1998). Gobernabilidad y políticas públicas. En R. Urzúa \& F. Agüero (Eds.), Fracturas en la gobernabilidad democrática (pp. 30-63). Santiago, Chile: Universidad de Chile. 